\title{
Adenosine and Kynurenic Acid Interactions: Possible Relevance for Schizophrenia Treatment?
}

\author{
Sarah Beggiato ${ }^{1 *}$, Mariachiara Zuccarini ${ }^{1}$, Tommaso Cassano ${ }^{2}$,

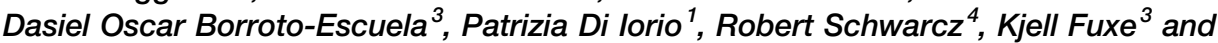 \\ Luca Ferraro ${ }^{5}$
}

${ }^{1}$ Department of Medical, Oral and Biotechnological Sciences, University of Chieti-Pescara, Chieti, Italy, ${ }^{2}$ Department of Medical and Surgical Sciences, University of Foggia, Foggia, Italy, ${ }^{3}$ Department of Neuroscience, Karolinska Institutet, Stockholm, Sweden, ${ }^{4}$ Department of Psychiatry, Maryland Psychiatric Research Center, University of Maryland School of Medicine, Baltimore, MD, United States, ${ }^{5}$ Department of Life Sciences and Biotechnology and LTTA Center, University of Ferrara, Ferrara, Italy

Keywords: adenosine $A_{1}$ and $A_{2 A}$ receptors, cognition, combinatory therapy, kynurenine pathway, receptor heteromers

\section{INTRODUCTION}

Schizophrenia is a severe and chronic mental disorder, mainly characterized by the presence of the so-called "positive" (delusions, hallucinations, disorganized thinking) and "negative" (anhedonia, blunted affect, social withdrawal) symptoms, as well as cognitive dysfunctions. Although several interrelated causes have been associated with the development of the pathology, it is generally accepted that the hyperfunction of dopaminergic and/or hypofunction of glutamatergic transmission (i.e., the so-called "combined glutamate/dopamine hypothesis of schizophrenia") might underlie the symptoms of schizophrenia (Howes et al., 2015; Snyder and Gao, 2020). Clinical indications demonstrate that positive symptoms respond well to conventional antipsychotic medications, which mainly act as dopamine $D_{2}$ receptor $\left(D_{2} R\right)$ antagonists, while negative symptoms and cognitive impairments are more difficult to be counteracted. Several non- $\mathrm{D}_{2} \mathrm{R}$ related mechanisms of action of antipsychotic drugs have been proposed over the last decades, but none has conclusively been proven effective. Furthermore, while the newer antipsychotic drugs produce fewer motor side effects than conventional "first generation" drugs, safety and tolerability concerns about weight gain and endocrinopathies often limit their use (Li et al., 2016). Thus, there is an urgent necessity for more effective and better-tolerated antipsychotic drugs, as well as to identify new molecular targets and develop mechanistically novel compounds that can address the various symptom dimensions of schizophrenia. Due to the complexity of the pathology, it seems likely, however, that a multitarget strategy, i.e., the use of multifunctional drugs or a combination of drugs affecting distinct targets, will lead to more effective therapeutic approaches.

Based on this background and recent findings, the present opinion paper was conceived to critically review possible interactions between adenosine and kynurenic acid (KYNA) in this context. These two neuromodulators may be pathophysiologically associated with schizophrenia, and a deeper understanding of their interactions may lead to the development of innovative strategies for the treatment of schizophrenia.

\section{Adenosine and Schizophrenia}

It is well recognized that, beside dopamine and glutamate systems, the purinergic system may be also involved in the pathophysiology of schizophrenia (Lara and Souza, 2000; Krügel, 2016; Cheffer et al., 2018). In fact, the so-called "adenosine hypothesis of schizophrenia" (Lara et al., 2006; Boison et al., 
2012; Hirota and Kishi, 2013; Rial et al., 2014) postulates that a reduced adenosine tone is involved in the dysregulation of glutamatergic and dopaminergic activity in schizophrenia patients. Accordingly, based on informative studies in experimental animals, adenosine receptor agonists may act as atypical antipsychotic drugs (Krügel, 2016).

Adenosine $\mathrm{A}_{2 \mathrm{~A}}$ receptors $\left(\mathrm{A}_{2 \mathrm{~A}} \mathrm{Rs}\right)$, which are highly expressed in the striatum and the olfactory tubercle, exert fine regulation of individual synapses (Hines and Haydon, 2014; Krügel, 2016), and their activation facilitates glutamate release and potentiates $\mathrm{N}$-methyl-D-aspartate (NMDA) receptor function. As a consequence, $\mathrm{A}_{2 \mathrm{~A}} \mathrm{Rs}$ regulate synaptic plasticity by promoting adequate (or aberrant) adaptive responses in neuronal circuits (Azdad et al., 2009; Boison and Aronica, 2015; Krügel, 2016). In general, adenosine and $\mathrm{A}_{2 \mathrm{~A}} \mathrm{R}$ agonists induce behavioral effects similar to those of dopamine receptor (DR) antagonists used as antipsychotics (Rimondini et al., 1997; Wardas, 2008; Shen et al., 2012; Borroto-Escuela et al., 2020). In fact, $\mathrm{A}_{2 \mathrm{~A}} \mathrm{R}$ agonists inhibit hyperlocomotion and sensorimotor gating deficits induced by DR agonists and/or NMDA receptor channel blockers in rodents (Krügel, 2016). More specifically, converging evidence suggests that heteroreceptor complexes containing $\mathrm{AR}$ and $\mathrm{DR}$ protomers, especially adenosine $\mathrm{A}_{2 \mathrm{~A}} \mathrm{R}-\mathrm{D}_{2} \mathrm{R}$ heteroreceptor complexes, exert strong inhibitory modulation of dorsal and ventral striato-pallidal GABA neurons (Ferrè et al., 1991; Fuxe et al., 2008; Borroto-Escuela et al., 2018; Borroto-Escuela et al., 2020). Thus, $A_{2 A} R$ agonists reduce $D_{2} R$ recognition and function by acting on the $\mathrm{A}_{2 \mathrm{~A}}-\mathrm{D}_{2}$ heteroreceptor complexes located in the dorsal and ventral striato-pallidal anti-reward GABA pathway. Upon activation of this pathway, the brain circuit involved increases the glutamate drive to the frontal cortex from the medial dorsal thalamic nucleus, and transfer of anti-reward information takes place (Fuxe et al., 2008; Borroto-Escuela et al., 2017; Borroto-Escuela et al., 2018; Borroto-Escuela et al., 2020). Thus, it was suggested more than a decade ago (Fuxe et al., 2008) and recently demonstrated (Borroto-Escuela et al., 2020; Valle-León et al., 2020) that drugs promoting $A_{2 A} R-D_{2} R$ heteromer formation might constitute an alternative strategy for the treatment of schizophrenia. Furthermore, $\mathrm{A}_{2 \mathrm{~A}} \mathrm{R}$ agonists can allow a reduction of the dose of the $\mathrm{D}_{2} \mathrm{R}$ antagonists which should reduce the side effects of classical and atypical antipsychotic drugs. These findings moved $\mathrm{A}_{2 \mathrm{~A}} \mathrm{R}$ agonists into the focus of interest for adenosinergic therapeutic options in the disease.

The adenosine $A_{1}$ receptor $\left(A_{1} R\right)$, too, has been proposed as a potential antipsychotic drug target (Ossowska et al., 2020). $A_{1} R s$ are coupled to the $G_{i / o}$ family of $G$-proteins, are abundantly present throughout the central nervous system, and appear to generally exert an inhibitory and neuroprotective 'tone' (Chen et al., 2014; Krügel, 2016). Activation of presynaptic $A_{1}$ Rs inhibits the release of neurotransmitters (e.g., glutamate, GABA, dopamine, serotonin and acetylcholine) and depresses postsynaptic neuronal signaling by inducing hyperpolarization (Paul et al., 2011). Notably, pre- and post-synaptic $A_{1} R$ activation, leading to reduced glutamate and GABA release as well as impaired NMDA receptor and $D_{1} R$ function, respectively, plays a major role in the "adenosine hypothesis" of schizophrenia (Fuxe et al., 2008; Krügel, 2016). Thus, as the pathophysiologically significant NMDA receptor hypofunction in the disease can be traced mainly to fast-spiking GABA neurons (Nakazawa and Sapkota, 2020), a reduction of $A_{1} R$ signaling should benefit critical neuronal circuits and consequently have positive effects on schizophrenia symptoms. In line with this view, $\mathrm{A}_{2 \mathrm{~A}} \mathrm{R}$ agonists might exert part of their antipsychotic action by activating the $\mathrm{A}_{2 \mathrm{~A}} \mathrm{R}$ protomer in a prejunctional $\mathrm{A}_{1}-\mathrm{A}_{2 \mathrm{~A}}$ receptor complex. Through this antagonistic receptor-receptor interaction, $A_{2 A} R$ agonists could lower the affinity of the $A_{1} R$ protomer and thus the inhibitory action of the $A_{1} R$ protomer on glutamate release (Ciruela et al., 2006; Franco et al., 2008; Borroto-Escuela et al., 2020). Antagonists of $A_{1} R$ receptors have indeed been shown to reduce memory impairment in experimental animals (Boison et al., 2012).

On the other hand, since activation of $A_{1}$ Rs on dopaminergic nerve terminals inhibits dopamine release (Paul et al., 2011; Zhang and Sulzer, 2012), $\mathrm{A}_{1} \mathrm{R}$ agonists, too, may counteract schizophrenia symptoms. In fact, preclinical findings have indicated that stimulation of $\mathrm{A}_{1} \mathrm{Rs}$ may have antipsychotic effects, although cognitive dysfunctions must be expected to be associated with the treatment (Ossowska et al., 2020). Specifically, recent studies demonstrated that the selective $\mathrm{A}_{1} \mathrm{R}$ agonist 5-Chloro-5'-deoxy-N6-( \pm )-(endo-norborn-2-yl) adenosine $\left(5^{\prime}-\mathrm{Cl}-5^{\prime}\right.$-deoxy-ENBA) reduces the hyperlocomotion caused by amphetamine or the non-competitive NMDA receptor antagonist dizolcipine (MK-801; Eyjolfsson et al., 2006; Ossowska et al., 2020). Inhibition of amphetamine- and MK-801-mediated hyperlocomotion may also be caused by allosteric interaction of $D_{1} R$ signaling in the $A_{1} R-D_{1} R$ heteroreceptor complex, which is located in striato-nigral and striato-entopeduncular GABA neurons as well as in $\mathrm{D}_{1} \mathrm{R}$-rich GABA neurons in the nucleus accumbens (Rimondini et al., 1997; Fuxe et al., 2007; Fuxe et al., 2008; Fuxe et al., 2020; Franco et al., 2008; Pérez-dela-Mora et al., 2020).

\section{Kynurenic Acid and Schizophrenia}

KYNA, an astrocyte-derived neuromodulator, has been repeatedly linked to the cognitive deficits that are observed in individuals with schizophrenia. KYNA is a metabolite of the kynurenine pathway (KP), which accounts for more than $90 \%$ of the degradation of the essential amino acid tryptophan (Cervenka et al., 2017). Through a series of enzymatic steps, the evolutionarily preserved KP generates not only KYNA but also a considerable number of other biologically active compounds, several of which play increasingly appreciated roles in brain physiology and pathology (Schwarcz et al., 2012). KYNA is produced directly from the pivotal KP metabolite kynurenine, either by oxidation (Ramos-Chávez 
et al., 2018) or by irreversible transamination by kynurenine aminotransferases (KATs; Guidetti et al., 2007). These enzymes are preferentially localized in astrocytes, which promptly release newly formed KYNA into the extracellular compartment (Turski et al., 1989; Guidetti et al., 2007). Though other molecular targets may be of relevance as well, the neurobiological effects of endogenous KYNA are mediated primarily through its actions as an antagonist of both the NMDA and the $a 7 \mathrm{nAChR}$ function, i.e. two receptors that are critically involved in cognitive processes (Moroni et al., 2012; Stone et al., 2013; Phenis et al., 2020). Consequently, as shown consistently in experimental animals, elevated brain KYNA levels are associated with a number of cognitive deficits, such as impairments in contextual learning and memory and abnormal visuospatial working memory (Schwarcz et al., 2012; Muneer, 2020). These effects are likely related to the fact that even relatively small fluctuations in KYNA levels bi-directionally affect the extracellular levels of neurotransmitters that play major roles in cognitive functions, including dopamine, acetylcholine, glutamate and GABA (Wu et al., 2007; Zmarowski et al., 2009; Konradsson-Geuken et al., 2010; Beggiato et al., 2013). Notably, selective pharmacological inhibition of KYNA formation has been shown to have procognitive effects in several established animal models (Kozak et al., 2014; Pocivavsek et al., 2019).

The observation that KYNA concentrations are significantly elevated in cortical brain regions and cerebrospinal fluid of individuals afflicted with schizophrenia (Erhardt et al., 2001; Schwarcz et al., 2001; Nilsson et al., 2005; Sathyasaikumar et al., 2011; Linderholm et al., 2012) raised the possibility that KYNA may be causally involved in the cognitive dysfunctions seen in these patients (cf. reviews by Wonodi and Schwarcz, 2010; Erhardt et al., 2017; Plitman et al., 2017; Muneer, 2020). This hypothesis is compatible with the fact that the expression of KYNA's key biological targets (i.e., NMDA receptors and a $7 \mathrm{nAChRs)}$ was found to be reduced in the brain of patients with schizophrenia (Guan et al., 1999; Young and Geyer, 2013; $\mathrm{Hu}$ et al., 2015). Together with the insights gained from the preclinical studies, these findings suggest that interventions leading to a decrease in brain KYNA may constitute a useful strategy for effecting cognitive improvement in the clinical population.

\section{Adenosine and Kynurenic Acid Interactions: Are They Relevant for Schizophrenia Treatments?}

Although neurobiological properties of adenosine may be linked to KYNA, interactions between the adenosine system and the KP have not been carefully examined so far. However, in an in vivo microdialysis study performed in rats, local perfusion of adenosine was shown to rapidly and concentrationdependently raise extracellular KYNA levels in the striatum (Wu et al., 2004). Interestingly, this effect was mimicked by perfusion of the $\mathrm{A}_{1} \mathrm{R}$ agonist $N^{6}$-cyclopentyladenosine (CPA), whereas the selective $\mathrm{A}_{2 \mathrm{~A}} \mathrm{R}$ agonist 2-p-(2-carboxyethyl) phenylethylamino- $5^{\prime}$-N-ethylcarboxamidoadenosine hydrochloride (CGS-21680) was ineffective. Furthermore, local perfusion of the $\mathrm{A}_{1} \mathrm{R}$ antagonist 8-cyclopentyltheophylline (CPT) attenuated the effect of adenosine on extracellular KYNA levels. As the effect of adenosine on KYNA was not observed in the excitotoxically lesioned, i.e., neuron-depleted, striatum, it appears that neuronal $A_{1} R$ activation influences glial KYNA synthesis indirectly (Wu et al., 2004).

While $\mathrm{A}_{2 \mathrm{~A}} \mathrm{R}$ activation does not appear to affect KYNA levels in the brain under physiological conditions, it is noteworthy that $\mathrm{A}_{2 \mathrm{~A}}$ Rs not only interact physically with $\mathrm{D}_{2}$ Rs (see above) but also with the NMDAR (Agnati et al., 2005; Liu et al., 2006). An $\mathrm{A}_{2 \mathrm{~A}} \mathrm{R}$ agonist may therefore inhibit the activity of the $\mathrm{D}_{2} \mathrm{R}$ protomer both in the $\mathrm{A}_{2 \mathrm{~A}} \mathrm{R}-\mathrm{D}_{2} \mathrm{R}$ heteromer (Borroto-Escuela and Fuxe, 2019) and in a putative $A_{2 A} R-D_{2} R-N M D A R$ heteromer, and thereby indirectly enhance NMDAR activity. By this mechanism, $\mathrm{A}_{2 \mathrm{~A}} \mathrm{R}$ stimulation could counteract and reduce the cognitive dysfunction caused by the elevated brain levels of the endogenous NMDAR antagonist KYNA in pathological situations (e.g., schizophrenia).

Furthermore, based on the postulated action of $\mathrm{A}_{2 \mathrm{~A}} \mathrm{R}$ agonists on prejunctional $A_{1}-A_{2 A}$ heteroreceptor complexes (Ciruela et al., 2006), it also seems possible that $A_{2 A} R$ agonists, in addition to inhibition of $\mathrm{D}_{2} \mathrm{R}$ signaling, cause a reduction in KYNA levels by allosteric inhibition of $\mathrm{A}_{1} \mathrm{R}$ signaling. In view of the study of $\mathrm{Wu}$ et al. (2004); see above), this mechanism, too, may only operate under pathological conditions.

Taken together, these phenomena may have implications for the proposed use of adenosine receptor agonists in the treatment of schizophrenia (Borroto-Escuela et al., 2020). Thus, the beneficial antipsychotic effects of $\mathrm{A}_{1} \mathrm{R}$ agonists, which are predicted from studies in experimental animals (Boison et al., 2012; Ossowska et al., 2020), may also result in cognitive deficits due to a $\mathrm{A}_{1} \mathrm{R}$-induced increase in KYNA levels. Co-treatment with drugs that are able to reduce brain KYNA levels may therefore ameliorate the untoward side effects of $A_{1} R$ agonists. Inhibitors of kynurenine aminotransferase II (KAT II), the principal enzyme responsible for the synthesis of rapidly mobilizable KYNA in the mammalian brain (Guidetti et al., 2007), deserve particular attention in this context (Rossi et al., 2010; Nematollahi et al., 2016; Plitman et al., 2017; Blanco-Ayala et al., 2020). Notably, the beneficial effects of KAT II inhibitors may be further enhanced by $\mathrm{A}_{2 \mathrm{~A}} \mathrm{R}$ agonists and may also improve negative symptoms in schizophrenia patients via allosteric inhibition of $\mathrm{D}_{2} \mathrm{R}$ signaling in $\mathrm{A}_{2 \mathrm{~A}} \mathrm{R}-\mathrm{D}_{2} \mathrm{R}$ heteroreceptor complexes of ventral striatal-pallidal GABA neurons (BorrotoEscuela et al., 2020).

\section{CONCLUSION}

The considerations outlined here indicate a possible relevance of adenosine and KYNA interactions in the pathophysiology and treatment of schizophrenia, and emphasize the need to 
investigate this issue in detail in future preclinical studies. Specifically, the effects of combined approaches with adenosine receptor ligands and compounds able to reduce brain KYNA levels (e.g., KAT II inhibitors) have not been assessed experimentally so far. Hypothesis testing in rats that were prenatally exposed to kynurenine, which have deficits resembling several of the cognitive impairments seen in schizophrenia patients (Hahn et al., 2018), may be particularly informative for this purpose. These studies may support the development of new multi-target therapeutic strategies that focus on both the purinergic system, especially in relation to adenosine receptor containing heteroreceptor complexes, and brain KYNA function.

\section{REFERENCES}

Agnati, L. F., Ferré, S., Burioni, R., Woods, A., Genedani, S., Franco, R., et al. (2005). Existence and theoretical aspects of homomeric and heteromeric dopamine receptor complexes and their relevance for neurological diseases. Neuromolec. Med. 7, 61-78. doi:10.1385/NMM:7:1-2:061

Azdad, K., Gall, D., Woods, A. S., Ledent, C., Ferré, S., and Schiffmann, S. N. (2009). Dopamine D2 and adenosine A2A receptors regulate NMDAmediated excitation in accumbens neurons through A2A-D2 receptor heteromerization. Neuropsychopharmacology 34, 972-986. doi:10.1038/ npp.2008.144

Beggiato, S., Antonelli, T., Tomasini, M. C., Tanganelli, S., Fuxe, K., Schwarcz, R., et al. (2013). Kynurenic acid, by targeting $\alpha 7$ nicotinic acetylcholine receptors, modulates extracellular GABA levels in the rat striatum in vivo. Eur. J. Neurosci. 37, 1470-1477. doi:10.1111/ejn.12160

Blanco-Ayala, T., Sathyasaikumar, K. V., Uys, J. D., Pérez-de-la-Cruz, V., Pidugu, L. S., and Schwarcz, R. (2020). N-Acetylcysteine inhibits kynurenine aminotransferase II. Neuroscience 444, 160-169. doi:10.1016/j.neuroscience.2020.07.049

Boison, D., and Aronica, E. (2015). Comorbidities in neurology: is adenosine the common link?. Neuropharmacology 97, 18-34. doi:10.1016/j.neuropharm.2015.04.031

Boison, D., Singer, P., Shen, H.-Y., Feldon, J., and Yee, B. K. (2012). Adenosine hypothesis of schizophrenia - opportunities for pharmacotherapy. Neuropharmacology 62, 1527-1543. doi:10.1016/j.neuropharm.2011.01.048

Borroto-Escuela, D. O., Carlsson, J., Ambrogini, P., Narváez, M., Wydra, K., Tarakanov, A. O., et al. (2017). Understanding the role of GPCR heteroreceptor complexes in modulating the brain networks in health and disease. Front. Cell Neurosci. 11, 37. doi:10.3389/fncel.2017.00037

Borroto-Escuela, D. O., Ferraro, L., Narvaez, M., Tanganelli, S., Beggiato, S., Liu, F., et al. (2020). Multiple adenosine-dopamine (A2A-D2 Like) heteroreceptor complexes in the brain and their role in schizophrenia. Cells 9, 1077. doi:10. 3390/cells9051077

Borroto-Escuela, D. O., and Fuxe, K. (2019). Adenosine heteroreceptor complexes in the basal ganglia are implicated in Parkinson's disease and its treatment. J. Neural Transm. 126, 455-471. doi:10.1007/s00702-019-01969-2

Borroto-Escuela, D. O., Wydra, K., Filip, M., and Fuxe, K. (2018). A2AR-D2R heteroreceptor complexes in cocaine reward and addiction. Trends Pharmacol. Sci. 39, 1008-1020. doi:10.1016/j.tips.2018.10.007

Cervenka, I., Agudelo, L. Z., and Ruas, J. L. (2017). Kynurenines: tryptophan's metabolites in exercise, inflammation, and mental health. Science 357 (6349), eaaf9794. doi:10.1126/science.aaf9794

Cheffer, A., Castillo, A. R. G., Corrêa-Velloso, J., Gonçalves, M. C. B., Naaldijk, Y., Nascimento, I. C., et al. (2018). Purinergic system in psychiatric diseases. Mol. Psychiatry 23, 94-106. doi:10.1038/mp.2017.188

Chen, J.-F., Lee, C.-f., and Chern, Y. (2014). Adenosine receptor neurobiology: overview. Int. Rev. Neurobiol. 119, 1-49. doi:10.1016/B978-0-12-801022-8. 00001-5

Ciruela, F., Casado, V., Rodrigues, R. J., Lujan, R., Burgueno, J., Canals, M., et al. (2006). Presynaptic control of striatal glutamatergic neurotransmission by adenosine A1-A2A receptor heteromers. J. Neurosci. 26, 2080-2087. doi:10. 1523/JNEUROSCI.3574-05.2006

\section{AUTHOR CONTRIBUTIONS}

LF, DOB-E, TC, and PD performed the literature review. MZ, KF, and RS helped to synthesize data and edited the text. SB reviewed the information and edited the text.

\section{FUNDING}

This work was supported by a grant from the University of Ferrara (FAR 2019) to LF, USPHS grant MH103222 (Silvio O. Conte Center for Translational Mental Health Research) to RS and a Swedish Research Council grant (04X-715, 2020-2022) to KF.

Erhardt, S., Blennow, K., Nordin, C., Skogh, E., Lindström, L. H., and Engberg, G. (2001). Kynurenic acid levels are elevated in the cerebrospinal fluid of patients with schizophrenia. Neurosci. Lett. 313, 96-98. doi:10.1016/s0304-3940(01)02242-x

Erhardt, S., Schwieler, L., Imbeault, S., and Engberg, G. (2017). The kynurenine pathway in schizophrenia and bipolar disorder. Neuropharmacology 112, 297-306. doi:10.1016/j.neuropharm.2016.05.020

Eyjolfsson, E. M., Brenner, E., Kondziella, D., and Sonnewald, U. (2006). Repeated injection of MK801: an animal model of schizophrenia? Neurochem. Int. 48, 541-546. doi:10.1016/j.neuint.2005.11.019

Ferré, S., Von Euler, G., Johansson, B., Fredholm, B. B., and Fuxe, K. (1991). Stimulation of high-affinity adenosine A2 receptors decreases the affinity of dopamine D2 receptors in rat striatal membranes. Proc. Natl. Acad. Sci. U.S.A. 88, 7238-7241. doi:10.1073/pnas.88.16.7238

Franco, R., Casadó, V., Cortés, A., Pérez-Capote, K., Mallol, J., Canela, E., et al. (2008). Novel pharmacological targets based on receptor heteromers. Brain Res. Rev. 58, 475-482. doi:10.1016/j.brainresrev.2008.06.002

Fuxe, K., Ferré, S., Genedani, S., Franco, R., and Agnati, L. F. (2007). Adenosine receptor-dopamine receptor interactions in the basal ganglia and their relevance for brain function. Physiol. Behav. 92, 210-217. doi:10.1016/j. physbeh.2007.05.034

Fuxe, K., Marcellino, D., Rivera, A., Diaz-Cabiale, Z., Filip, M., Gago, B., et al. (2008). Receptor-receptor interactions within receptor mosaics. Impact on neuropsychopharmacology. Brain Res. Rev. 58, 415-452. doi:10.1016/j. brainresrev.2007.11.007

Guan, Z.-Z., Zhang, X., Blennow, K., and Nordberg, A. (1999). Decreased protein level of nicotinic receptor $\alpha 7$ subunit in the frontal cortex from schizophrenic brain. Neuroreport 10, 1779-1782. doi:10.1097/00001756-199906030-00028

Guidetti, P., Hoffman, G. E., Melendez-Ferro, M., Albuquerque, E. X., and Schwarcz, R. (2007). Astrocytic localization of kynurenine aminotransferase II in the rat brain visualized by immunocytochemistry. Glia 55, 78-92. doi:10.1002/glia.20432

Hahn, B., Reneski, C. H., Pocivavsek, A., and Schwarcz, R. (2018). Prenatal kynurenine treatment in rats causes schizophrenia-like broad monitoring deficits in adulthood. Psychopharmacology 235, 651-661. doi:10.1007/ s00213-017-4780-9

Hines, D. J., and Haydon, P. G. (2014). Astrocytic adenosine: from synapses to psychiatric disorders. Phil. Trans. R. Soc. B 369, 20130594. doi:10.1098/rstb. 2013.0594

Hirota, T., and Kishi, T. (2013). Adenosine hypothesis in schizophrenia and bipolar disorder: a systematic review and meta-analysis of randomized controlled trial of adjuvant purinergic modulators. Schizophrenia Res. 149, 88-95. doi:10.1016/ j.schres.2013.06.038

Howes, O., McCutcheon, R., and Stone, J. (2015). Glutamate and dopamine in schizophrenia: an update for the 21st century. J. Psychopharmacol. 29, 97-115. doi:10.1177/0269881114563634

Hu, W., MacDonald, M. L., Elswick, D. E., and Sweet, R. A. (2015). The glutamate hypothesis of schizophrenia: evidence from human brain tissue studies. Ann. N.Y. Acad. Sci. 1338, 38. doi:10.1111/nyas.12547

Konradsson-Geuken, Å., Wu, H. Q., Gash, C. R., Alexander, K. S., Campbell, A., Sozeri, Y., et al. (2010). Cortical kynurenic acid bi-directionally modulates prefrontal glutamate levels as assessed by microdialysis and rapid electrochemistry. Neuroscience 169, 1848-1859. doi:10.1016/j.neuroscience.2010.05.052 
Kozak, R., Campbell, B. M., Strick, C. A., Horner, W., Hoffmann, W. E., Kiss, T., et al. (2014). Reduction of brain kynurenic acid improves cognitive function. J. Neurosci. 34, 10592-10602. doi:10.1523/JNEUROSCI.1107-14.2014

Krügel, U. (2016). Purinergic receptors in psychiatric disorders. Neuropharmacology 104, 212-225. doi:10.1016/j.neuropharm.2015.10.032

Lara, D. R., Dall'Igna, O. P., Ghisolfi, E. S., and Brunstein, M. G. (2006). Involvement of adenosine in the neurobiology of schizophrenia and its therapeutic implications. Prog. Neuro-Psychopharmacology Biol. Psychiatry 30, 617-629. doi:10.1016/j.pnpbp.2006.02.002

Lara, D. R., and Souza, D. O. (2000). Schizophrenia: a purinergic hypothesis. Med. Hypotheses 54, 157-166. doi:10.1054/mehy.1999.0003

Li, P., L. Snyder, G., and E. Vanover, K. (2016). Dopamine targeting drugs for the treatment of schizophrenia: past, present and future. Ctmc 16, 3385-3403. doi:10.2174/1568026616666160608084834

Linderholm, K. R., Skogh, E., Olsson, S. K., Dahl, M.-L., Holtze, M., Engberg, G., et al. (2012). Increased levels of kynurenine and kynurenic acid in the CSF of patients with schizophrenia. Schizophrenia Bull. 38, 426-432. doi:10.1093/schbul/sbq086

Liu, X.-Y., Chu, X.-P., Mao, L.-M., Wang, M., Lan, H.-X., Li, M.-H., et al. (2006). Modulation of D2R-NR2B interactions in response to cocaine. Neuron 52, 897-909. doi:10.1016/j.neuron.2006.10.011

Moroni, F., Cozzi, A., Sili, M., and Mannaioni, G. (2012). Kynurenic acid: a metabolite with multiple actions and multiple targets in brain and periphery. J. Neural Transm. 119, 133-139. doi:10.1007/s00702-011-0763-x

Muneer, A. (2020). Kynurenine pathway of tryptophan metabolism in neuropsychiatric disorders: pathophysiologic and therapeutic considerations. Clin. Psychopharmacol. Neurosci. 18, 507-526. doi:10.9758/cpn.2020.18.4.507

Nakazawa, K., and Sapkota, K. (2020). The origin of NMDA receptor hypofunction in schizophrenia. Pharmacol. Ther. 205, 107426. doi:10.1016/j.pharmthera. 2019.107426

Nematollahi, A., Sun, G., Jayawickrama, G., and Church, W. (2016). Kynurenine aminotransferase isozyme inhibitors: a review. Int. J. Mol. Sci. 17, 946. doi:10. 3390/ijms 17060946

Nilsson, L. K., Linderholm, K. R., Engberg, G., Paulson, L., Blennow, K., Lindström, L. H., et al. (2005). Elevated levels of kynurenic acid in the cerebrospinal fluid of male patients with schizophrenia. Schizophrenia Res. 80, 315-322. doi:10.1016/ j.schres.2005.07.013

Ossowska, K., Kosmowska, B., and Wardas, J. (2020). Potential antipsychotic action of the selective agonist of adenosine A1 receptors, $5^{\prime}$-Cl-5'-deoxy-ENBA, in amphetamine and MK-801 rat models. Pharmacol. Rep. 72, 580-588. doi:10. 1007/s43440-020-00093-3

Paul, S., Elsinga, P. H., Ishiwata, K., Dierckx, R. A., and van Waarde, A. (2011). Adenosine A1 receptors in the central nervous system: their functions in health and disease, and possible elucidation by PET imaging. Curr. Med. Chem. 18, 4820. doi:10.2174/092986711797535335

Pérez de la Mora, M., Hernandez-Mondragon, C., Crespo-Ramirez, M., RejonOrantes, J., Borroto-Escuela, D. O., and Fuxe, K. (2020). Conventional and novel pharmacological approaches to treat dopamine-related disorders: focus on Parkinson's disease and schizophrenia. Neuroscience 439, 301-318. doi:10. 1016/j.neuroscience.2019.07.026

Phenis, D., Vunck, S. A., Valentini, V., Arias, H., Schwarcz, R., and Bruno, J. P. (2020). Activation of alpha7 nicotinic and NMDA receptors is necessary for performance in a working memory task. Psychopharmacology 237, 1723-1735. doi:10.1007/s00213-020-05495-y

Plitman, E., Iwata, Y., Caravaggio, F., Nakajima, S., Chung, J. K., Gerretsen, P., et al. (2017). Kynurenic acid in schizophrenia: a systematic review and meta-analysis. Schizophr Bull. 43, 764-777. doi:10.1093/schbul/sbw221

Pocivavsek, A., Elmer, G. I., and Schwarcz, R. (2019). Inhibition of kynurenine aminotransferase II attenuates hippocampus-dependent memory deficit in adult rats treated prenatally with kynurenine. Hippocampus 29, 73-77. doi:10.1002/hipo.23040

Ramos-Chávez, L. A., Lugo Huitrón, R., González Esquivel, D., Pineda, B., Ríos, C., Silva-Adaya, D., et al. (2018). Relevance of alternative routes of kynurenic acid production in the brain. Oxid. Med. Cell. Longev. 2018, 1. doi:10.1155/2018/5272741

Rial, D., Lara, D. R., and Cunha, R. A. (2014). The adenosine neuromodulation system in schizophrenia. Int. Rev. Neurobiol. 119, 395-449. doi:10.1016/B978$0-12-801022-8.00016-7$
Rimondini, R., Ferré, S., Ögren, S. O., and Fuxe, K. (1997). Adenosine A2A agonists: a potential new type of atypical antipsychotic. Neuropsychopharmacology 17, 82-91. doi:10.1016/S0893-133X(97)00033-X

Rossi, F., Valentina, C., Garavaglia, S., Sathyasaikumar, K. V., Schwarcz, R., Kojima, S.-I., et al. (2010). Crystal structure-based selective targeting of the pyridoxal $5^{\prime}$ phosphate dependent enzyme kynurenine aminotransferase II for cognitive enhancement. J. Med. Chem. 53, 5684-5689. doi:10.1021/jm100464k

Sathyasaikumar, K. V., Stachowski, E. K., Wonodi, I., Roberts, R. C., Rassoulpour, A., McMahon, R. P., et al. (2011). Impaired kynurenine pathway metabolism in the prefrontal cortex of individuals with schizophrenia. Schizophrenia Bull. 37, 1147-1156. doi:10.1093/schbul/sbq112

Schwarcz, R., Bruno, J. P., Muchowski, P. J., and Wu, H.-Q. (2012). Kynurenines in the mammalian brain: when physiology meets pathology. Nat. Rev. Neurosci. 13, 465-477. doi:10.1038/nrn3257

Schwarcz, R., Rassoulpour, A., Wu, H.-Q., Medoff, D., Tamminga, C. A., and Roberts, R. C. (2001). Increased cortical kynurenate content in schizophrenia. Biol. Psychiatry 50, 521-530. doi:10.1016/s0006-3223(01)01078-2

Shen, H.-Y., Singer, P., Lytle, N., Wei, C. J., Lan, J.-Q., Williams-Karnesky, R. L., et al. (2012). Adenosine augmentation ameliorates psychotic and cognitive endophenotypes of schizophrenia. J. Clin. Invest. 122, 2567-2577. doi:10.1172/JCI62378

Snyder, M. A., and Gao, W.-J. (2020). NMDA receptor hypofunction for schizophrenia revisited: perspectives from epigenetic mechanisms. Schizophrenia Res. 217, 60-70. doi:10.1016/j.schres.2019.03.010

Stone, T. W., Stoy, N., and Darlington, L. G. (2013). An expanding range of targets for kynurenine metabolites of tryptophan. Trends Pharmacol. Sci. 34, 136-143. doi:10.1016/j.tips.2012.09.006

Turski, W. A., Gramsbergen, J. B. P., Traitler, H., and Schwarcz, R. (1989). Rat brain slices produce and liberate kynurenic acid upon exposure to L-kynurenine. J. Neurochem. 52, 1629-1636. doi:10.1111/j.1471-4159.1989.tb09218.x

Valle-León, M., Callado, L. F., Aso, E., Cajiao-Manrique, M. M., Sahlholm, K., López-Cano, M., et al. (2020). Decreased striatal adenosine A2A-dopamine D2 receptor heteromerization in schizophrenia. Neuropsychopharmacology 46, 665-672. doi:10.1038/s41386-020-00872-9

Wardas, J. (2008). Potential role of adenosine A2A receptors in the treatment of schizophrenia. Front. Biosci. 13, 4071-4096. doi:10.2741/2995

Wonodi, I., and Schwarcz, R. (2010). Cortical kynurenine pathway metabolism: a novel target for cognitive enhancement in Schizophrenia. Schizophrenia Bull. 36, 211-218. doi:10.1093/schbul/sbq002

Wu, H.-Q., Fuxe, K., and Schwarcz, R. (2004). Neuronal A1 receptors mediate increase in extracellular kynurenic acid after local intrastriatal adenosine infusion. J. Neurochem. 90, 621-628. doi:10.1111/j.1471-4159.2004.02531.x

Wu, H.-Q., Rassoulpour, A., and Schwarcz, R. (2007). Kynurenic acid leads, dopamine follows: a new case of volume transmission in the brain? J. Neural Transm. 114, 33-41. doi:10.1007/s00702-006-0562-y

Young, J. W., and Geyer, M. A. (2013). Evaluating the role of the alpha-7 nicotinic acetylcholine receptor in the pathophysiology and treatment of schizophrenia. Biochem. Pharmacol. 86, 1122-1132. doi:10.1016/j.bcp.2013.06.031

Zhang, H., and Sulzer, D. (2012). Regulation of striatal dopamine release by presynaptic auto- and heteroreceptors. Basal Ganglia 2, 5-13. doi:10.1016/j. baga.2011.11.004

Zmarowski, A., Wu, H.-Q., Brooks, J. M., Potter, M. C., Pellicciari, R., Schwarcz, R., et al. (2009). Astrocyte-derived kynurenic acid modulates basal and evoked cortical acetylcholine release. Eur. J. Neurosci. 29, 529-538. doi:10.1111/j.14609568.2008.06594.x

Conflict of Interest: The authors declare that the research was conducted in the absence of any commercial or financial relationships that could be construed as a potential conflict of interest.

Copyright (c) 2021 Beggiato, Zuccarini, Cassano, Borroto-Escuela, Di Iorio, Schwarcz, Fuxe and Ferraro. This is an open-access article distributed under the terms of the Creative Commons Attribution License (CC BY). The use, distribution or reproduction in other forums is permitted, provided the original author(s) and the copyright owner(s) are credited and that the original publication in this journal is cited, in accordance with accepted academic practice. No use, distribution or reproduction is permitted which does not comply with these terms. 